follow-up. If surgery is performed, it should be limited to a biopsy or decompression. (Steinbok P, Hentschel S, Almqvist P, Cochrane DD, Poskitt K. Management of optic chiasmatic/hypothalamic astrocytomas in children. Can I Neurol Sci May 2002;29:132-138). (Reprints: Dr Paul Steinbok, Division of Pediatric Neurosurgery, British Columbia's Children's Hospital, 4480 Oak Street, A323, Vancouver, BC, V6H 3V4, Canada).

COMMENT. Optic chiasmatic and optic chiasmatic/hypothalamic tumors are different in their clinical presentation and behavior. Neurofibromatosis I is a feature of only the optic chiasmatic tumors. Optic chiasmatic tumors are managed by observation and treatment is required mainly for associated non-optic tumors. In chiasmatic/hypothalamic tumors, radical resection should be avoided. Although radiotherapy is effective in preventing progression, it should be delayed as long as posible because of the risk of long-term complications. Chemotherapy as the first line of treatment for optic/hypothalamic tumors may delay the need for radiotherapy but it may not be effective in preventing progression.

\title{
COGNITIVE IMPAIRMENT FOLLOWING INTRATHECAL METHOTREXATE FOR MEDULLOBLASTOMA
}

Intelligence, executive function, attention, visual perception, and shortterm memory were assessed in two groups of children who underwent surgery and subsequent radiotherapy and chemotherapy for cerebellar medulloblastoma at the Carlo Besta National Neurologic Institute, Milan, Italy. All 21 patients selected received the same combined radiotherapy-chemotherapy, but only one group (11 patients) was treated with intrathecal methotrexate (MTX) in addition. Both groups performed worse on cognitive tests than matched controls (cousins and siblings). Children younger than 10 years receiving MTX had significantly lower scores in all tests, and particularly executive function. Their impaired performance on arithmetic, comprehension, and block design was directly correlated with the extent of periventriculat leukomalacia observed on MRI. Only short-term memory was impaired in the MTX patients older than 10 years. In the group not receiving MTX, the 3 to 10 year old patients did significantly worse than controls in three tests only, whereas patients older than 10 showed no impairment in test performance compared to controls. The use of intrathecal methotrexate in the treatment of medulloblastoma should be reassessed. (Riva D, Giorgi C, Nichelli $F$ et al. Intrathecal methotrexate affects cognitive function in children with medulloblastoma. Neurology July (1 of 2) 2002;59:48-53). (Reprints: Dr Daria Riva, Divisione di Neurologia dello Sviluppo, Instituto Nazionale Neurologico, Via Celeria, 11, 20133 Milano, Italy).

COMMENT. Intrathecal methotrexate therapy in children with medulloblastoma may worsen the cognitive deficits induced by chemotherapy and radiotherapy. Children younger than 10 years are especially at risk of impaired cognitive function following methotrexate.

The increase in survival rate of children with brain tumors over the past 20 years has been accompanied by a decrease in intellectual functioning and other complications. (Duffner PK, Cohen ME. 1991; see Progress in Pediatric Neurology II, PNB Publ, 1994;pp339-341). The omission of methotrexate may avoid the complications of leukoencephalopathy and dementia. Complications of cranial irradiation are reviewed in Progress in Pediatric Neurology III, 1997;pp423-430. 